\title{
Large time behavior for the fractional Ginzburg-Landau equations near the BCS-BEC crossover regime of Fermi gases
}

Lang $\mathrm{Li}^{\dagger}$, Lingyu Jin ${ }^{\dagger}$ and Shaomei Fang*

\section{"Correspondence:} dz90@scau.edu.cn

Department of Mathematics, South China Agricultural University,

Wushan Road, Guangzhou, 510642, China

${ }^{\dagger}$ Equal contributors

\section{然 Springer}

\begin{abstract}
In this paper, we consider the fractional Ginzburg-Landau equations near the Bardeen-Cooper-Schrieffer-Bose-Einstein-condensate (BCS-BEC) crossover of atomic Fermi gases. This fractional Ginzburg-Landau equations can be viewed as a generalization of the integral differential equations proposed by Machida and Koyama (Phys. Rev. A 74:033603, 2006). By using the Galerkin method and a priori estimates, together with the properties of Sobolev spaces, we first establish the existence and uniqueness of weak solutions to these equations and then we prove the existence of global attractors.
\end{abstract}

Keywords: fractional Ginzburg-Landau equations; global attractors; weak solution

\section{Introduction}

In this paper, we consider the fractional Ginzburg-Landau equations for atomic Fermi gases near the BCS-BEC crossover as follows:

$$
\begin{aligned}
& -i d u_{t}=\left(-\frac{d g^{2}+1}{U}+a\right) u+g[a+d(2 v-2 \mu)] \varphi \\
& \quad-\frac{c}{4 m} \Lambda^{2 \alpha} u-\frac{g}{4 m}(c-d) \Lambda^{2 \alpha} \varphi-b|u+g \varphi|^{2}(u+g \varphi)-i d f(x), \\
& i \varphi_{t}=-i \beta \varphi-\frac{g}{U} u+(2 v-2 \mu) \varphi+\frac{1}{4 m} \Lambda^{2 \alpha} \varphi+i h(x), \\
& u(x, 0)=u_{0}(x), \quad \varphi(x, 0)=\varphi_{0}(x), \quad x \in \mathbb{R}^{n}, \\
& u\left(x+2 \pi e_{i}, t\right)=u(x, t), \quad \varphi\left(x+2 \pi e_{i}, t\right)=\varphi(x, t), \quad x \in \mathbb{R}^{n}, t \geq 0,
\end{aligned}
$$

where $u(x, t)$ is the fermion-pair field and $\varphi(x, t)$ is the condensed boson field, $\mu$ is the chemical potential, $2 v$ is the threshold energy of the Feshbach resonance, $g$ is the coupling constant describes the process arising from the Feshbach resonance, in which a boson is created from two fermionic atoms and vice versa. $d$ is generally complex, in the BCS limit $d$ can be considered to be pure imaginary, while in the BEC region, the imaginary part of $d$ vanished. $U>0, a, b, c, \beta$ are real coefficients, the square root of the Laplacian, $\Lambda=(-\Delta)^{\frac{1}{2}}$, is the so-called Zygmund operator. In this paper, we restrict ourselves to the fractional order $\alpha \in\left(\frac{1}{2}, 1\right]$ and the space dimension $n=1$.

(c) The Author(s) 2017. This article is distributed under the terms of the Creative Commons Attribution 4.0 International License (http://creativecommons.org/licenses/by/4.0/), which permits unrestricted use, distribution, and reproduction in any medium, provided you give appropriate credit to the original author(s) and the source, provide a link to the Creative Commons license, and indicate if changes were made. 
The BCS-BEC crossover phenomenon has been experimentally realized by using ultracold gases of ${ }^{6} \mathrm{Li}$ and ${ }^{40} \mathrm{~K}$ atoms and it has opened a new era for the study of some longstanding theoretical proposals in many fermion systems. Since the conventional perturbation theory is no longer valid, the equation of state and dynamic properties of the BCS-BEC crossover have become a big challenge for quantum theory. In recent years, a number of groups have studied the atomic Fermi gases from various views which help us to deeply understand the physics of the pseudogap and Berezinskii-Kosterlitz-Thouless transitions in fermionic systems.

In 2006, on the basis of the functional integral formalism, Machida and Koyama [1] constructed a time-dependent Ginzburg-Landau theory for the superfluid atomic Fermi gases near the Feshbach resonance from the fermion-boson model. The time-dependent Ginzburg-Landau theory just can be described as (1)-(2). In the case of $g=0, \alpha=1$, (1)(2) are decoupled and reduced to the conventional time-dependent Ginzburg-Landau (TDGL) equation and the linearized Gross-Pitaevskii (GP) equation, which intensively were studied in the past decades. In the case of $g \neq 0, \alpha=1$, Chen and Guo [2-4] obtained the global existence and uniqueness of weak solutions to (1)-(2) with periodic boundary conditions, and then by the properties of Besov and Sobolev spaces and matrix theory, together with the energy method, they established the global existence of classical solutions. The existence of global attractors to (1)-(4) was proved by Fang, Jin and Guo [5]. In [6], Guo et al. considered the numerical solution of Ginzburg-Landau equations near the BCS-BEC crossover through finite difference method and analyzed its convergence and stability. In [7], the Hopf bifurcation of the above equations was studied and numerical simulations were also given.

In this paper, motivated by [8] and [9], we extend the result to fractional GinzburgLandau equations for atomic Fermi gases near the BCS-BEC crossover. When $\alpha$ is not an integer, the fractional dissipation operator $\Lambda^{2 \alpha}$ is nonlocal and can be regarded as the infinitesimal generators of Lévy stable diffusion processes. More and more researchers have found that fractional differential equations play an important role in mathematical physics and can be used to describe some physical phenomenon more exactly than integral differential equations. There have been extensive study of fractional differential equations including the fractional Ginzburg-Landau equation [8], the fractional Schrödinger equation [9], the fractional Landau-Lifshitz-Gilbert equation [10], the fractional Landau-Lifshitz equation [11] etc. For more details, see [12-15].

The rest of this paper is organized as follows. In the next section, we give some notations. In Section 3, we give a priori estimates. In Section 4, we prove the global existence of weak solutions to (1)-(4). Finally, the existence of global attractors is obtained.

\section{Notations}

Let $\Omega=[0,2 \pi], d=d_{r}+i d_{i}, m=m_{r}+i m_{i}$, and $|d|^{2}=d_{r}^{2}+d_{i}^{2},|m|^{2}=m_{r}^{2}+m_{i}^{2}$. Denote by $L^{p}(\Omega)$ the usual Sobolev space of the pth-power integrable functions normed by

$$
\|f\|_{L^{p}(\Omega)}=\left(\int_{\Omega}|f(x)|^{p} d x\right)^{\frac{1}{p}}, \quad\|f\|_{L^{\infty}(\Omega)}=\underset{\Omega}{\operatorname{ess} \sup }|f(x)| .
$$


When no confusion arises, we set $L^{p}:=L^{p}(\Omega)$ for $1 \leq p \leq \infty$. Similarly, we define the space $L^{p}(0, T ; X)$ with the norm

$$
\begin{aligned}
& \|f\|_{L^{p}(0, T ; X)}=\left(\int_{0}^{T}\|f\|_{X}^{p} d t\right)^{\frac{1}{p}}<\infty \quad(1 \leq p<\infty), \\
& \|f\|_{L^{\infty}(0, T ; X)}=\underset{0 \leq t \leq T}{\operatorname{ess} \sup }\|f\|_{X}<\infty .
\end{aligned}
$$

If $u$ is a periodic function, then it can be identified with the Fourier series

$$
u=\sum_{j \in \mathbb{Z}} a_{j} e^{i j \cdot x}
$$

Moreover, $\Lambda^{\alpha} u$ can be defined by

$$
\Lambda^{\alpha} u=\sum_{j \in \mathbb{Z}}|j|^{\alpha} a_{j} e^{i j \cdot x}
$$

Define

$$
E=\left\{\left.u\left|u=\sum_{j \in \mathbb{Z}} a_{j} e^{i j \cdot x}, \sum_{j \in \mathbb{Z}} a_{j}^{2}<\infty, \sum_{j \in \mathbb{Z}}\right| j\right|^{2 \alpha} a_{j}^{2}<\infty\right\},
$$

and $H^{\alpha}$ denotes a complete space of $E$ with the induced norm

$$
\|u\|_{H^{\alpha}}=\left(\sum_{j \in \mathbb{Z}} a_{j}^{2}\right)^{\frac{1}{2}}+\left(\sum_{j \in \mathbb{Z}}|j|^{2 \alpha} a_{j}^{2}\right)^{\frac{1}{2}} .
$$

Then $H^{\alpha}$ is a Banach space. It is easy to show that $H^{\alpha}$ is a Hilbert space with the inner product

$$
(u, v)_{H^{\alpha}}=\left(\Lambda^{\alpha} u, \Lambda^{\alpha} v\right)=\sum_{j \in \mathbb{Z}}|j|^{2 \alpha} a_{j} b_{j}
$$

where $\Lambda^{\alpha} v=\sum_{j \in \mathbb{Z}}|j|^{\alpha} b_{j} e^{i j \cdot x}$. For more details, see [9].

We denote positive constants by $C$; they may change from one line to the next line.

\section{A priori estimates}

Lemma 1 Assume that $u_{0} \in L^{2}(\Omega), \varphi_{0} \in L^{2}(\Omega), f(x) \in L^{2}(\Omega), h(x) \in L^{2}(\Omega)$. Let $(u, \varphi)$ be the solution to (1)-(4). Then, for any $0<T<\infty, U>0, c>0, m_{i} d_{r}+m_{r} d_{i}>0, b>0, m_{i}>0$, $d_{i}>0$, and $\beta>0$, we have

$$
\begin{aligned}
\| u & +g \varphi\left\|_{L^{2}}^{2}+\right\| \varphi\left\|_{L^{2}}^{2}+\int_{0}^{T}\right\| u+g \varphi \|_{L^{4}}^{4} d t \\
& +\int_{0}^{T}\left\|\Lambda^{\alpha}(u+g \varphi)\right\|_{L^{2}}^{2} d t+\int_{0}^{T}\left\|\Lambda^{\alpha} \varphi\right\|_{L^{2}}^{2} d t \leq C,
\end{aligned}
$$

where the constant $C$ depending on the initial data and $T$. 
Proof From (1)-(2), we have

$$
\begin{aligned}
(u+g \varphi)_{t}= & \frac{i a}{d}(u+g \varphi)-\frac{i}{d U}(u+g \varphi)+\frac{i g}{d U} \varphi-\beta g \varphi \\
& -\frac{i c}{4 m d} \Lambda^{2 \alpha}(u+g \varphi)-\frac{i b}{d}|u+g \varphi|^{2}(u+g \varphi)+(f+g h) .
\end{aligned}
$$

Multiplying (5) by $\overline{(u+g \varphi)}$, integrating over $\Omega$, and taking the real part, we have

$$
\begin{aligned}
\frac{1}{2} \frac{d}{d t}\|u+g \varphi\|_{L^{2}}^{2}= & \left(\frac{a d_{i}}{|d|^{2}}-\frac{d_{i}}{|d|^{2} U}\right)\|u+g \varphi\|_{L^{2}}^{2}+\operatorname{Re}\left(\frac{i g}{d U} \int_{\Omega} \varphi \overline{(u+g \varphi)} d x\right) \\
& -\operatorname{Re}\left(\beta g \int_{\Omega} \varphi \overline{(u+g \varphi)} d x\right)-\frac{c\left(m_{i} d_{r}+m_{r} d_{i}\right)}{4|m|^{2}|d|^{2}}\left\|\Lambda^{\alpha}(u+g \varphi)\right\|_{L^{2}}^{2} \\
& -\frac{b d_{i}}{|d|^{2}}\|u+g \varphi\|_{L^{4}}^{4}+\operatorname{Re}\left(\int_{\Omega}(f(x)+g h(x)) \overline{(u+g \varphi)} d x\right) .
\end{aligned}
$$

Multiplying (2) by $\bar{\varphi}$, integrating over $\Omega$, and taking the imaginary part, we have

$$
\begin{aligned}
\frac{1}{2} \frac{d}{d t}\|\varphi\|_{L^{2}}^{2}= & -\beta\|\varphi\|_{L^{2}}^{2}+\operatorname{Re}\left(\frac{i g}{U} \int_{\Omega}(u+g \varphi) \bar{\varphi} d x\right) \\
& -\frac{m_{i}}{4|m|^{2}}\left\|\Lambda^{\alpha} \varphi\right\|_{L^{2}}^{2}+\operatorname{Re}\left(\int_{\Omega} h(x) \bar{\varphi} d x\right)
\end{aligned}
$$

Combining the above two equations, we obtain

$$
\begin{aligned}
& \frac{1}{2} \frac{d}{d t}\left(\|u+g \varphi\|_{L^{2}}^{2}+\|\varphi\|_{L^{2}}^{2}\right) \\
& =\operatorname{Re}\left(\frac{i g}{d U} \int_{\Omega} \varphi \overline{(u+g \varphi)} d x\right)-\operatorname{Re}\left(\beta g \int_{\Omega} \varphi \overline{(u+g \varphi)} d x\right) \\
& +\operatorname{Re}\left(\int_{\Omega}(f(x)+g h(x)) \overline{(u+g \varphi)} d x\right)+\operatorname{Re}\left(\int_{\Omega} h(x) \bar{\varphi} d x\right) \\
& +\operatorname{Re}\left(\frac{i g}{U} \int_{\Omega}(u+g \varphi) \bar{\varphi} d x\right)+\left(\frac{a d_{i}}{|d|^{2}}-\frac{d_{i}}{|d|^{2} U}\right)\|u+g \varphi\|_{L^{2}}^{2} \\
& -\frac{c\left(m_{i} d_{r}+m_{r} d_{i}\right)}{4|m|^{2}|d|^{2}}\left\|\Lambda^{\alpha}(u+g \varphi)\right\|_{L^{2}}^{2}-\frac{b d_{i}}{|d|^{2}}\|u+g \varphi\|_{L^{4}}^{4} \\
& -\beta\|\varphi\|_{L^{2}}^{2}-\frac{m_{i}}{4|m|^{2}}\left\|\Lambda^{\alpha} \varphi\right\|_{L^{2}}^{2} \\
& \leq \frac{1}{2}\left|\frac{g}{d U}\right|\|u+g \varphi\|_{L^{2}}^{2}+\frac{1}{2}\left|\frac{g}{d U}\right|\|\varphi\|_{L^{2}}^{2}+\frac{1}{2}|\beta g|\|u+g \varphi\|_{L^{2}}^{2} \\
& +\frac{1}{2}|\beta g|\|\varphi\|_{L^{2}}^{2}+\frac{1}{2}\|u+g \varphi\|_{L^{2}}^{2}+\frac{1}{2}\left(\|f\|_{L^{2}}^{2}+g^{2}\|h\|_{L^{2}}^{2}\right) \\
& +\frac{1}{2}\|\varphi\|_{L^{2}}^{2}+\frac{1}{2}\|h\|_{L^{2}}^{2}+\frac{1}{2} \frac{|g|}{U}\|u+g \varphi\|_{L^{2}}^{2}+\frac{1}{2} \frac{|g|}{U}\|\varphi\|_{L^{2}}^{2} \\
& +\left(\frac{a d_{i}}{|d|^{2}}-\frac{d_{i}}{|d|^{2} U}\right)\|u+g \varphi\|_{L^{2}}^{2}-\frac{c\left(m_{i} d_{r}+m_{r} d_{i}\right)}{4|m|^{2}|d|^{2}}\left\|\Lambda^{\alpha}(u+g \varphi)\right\|_{L^{2}}^{2} \\
& -\frac{b d_{i}}{|d|^{2}}\|u+g \varphi\|_{L^{4}}^{4}-\beta\|\varphi\|_{L^{2}}^{2}-\frac{m_{i}}{4|m|^{2}}\left\|\Lambda^{\alpha} \varphi\right\|_{L^{2}}^{2}
\end{aligned}
$$




$$
\begin{aligned}
\leq & \frac{1}{2} C_{1}\left(\|u+g \varphi\|_{L^{2}}^{2}+\|\varphi\|_{L^{2}}^{2}\right)+\frac{1}{2}\|f\|_{L^{2}}^{2}+\frac{1}{2}\left(1+g^{2}\right)\|h\|_{L^{2}}^{2} \\
& -\frac{c\left(m_{i} d_{r}+m_{r} d_{i}\right)}{4|m|^{2}|d|^{2}}\left\|\Lambda^{\alpha}(u+g \varphi)\right\|_{L^{2}}^{2}-\frac{b d_{i}}{|d|^{2}}\|u+g \varphi\|_{L^{4}}^{4} \\
& -\beta\|\varphi\|_{L^{2}}^{2}-\frac{m_{i}}{4|m|^{2}}\left\|\Lambda^{\alpha} \varphi\right\|_{L^{2}}^{2},
\end{aligned}
$$

where $C_{1}=\max \left\{\left|\frac{g}{d U}\right|+\frac{|g|}{U}+|\beta g|+1,\left|\frac{g}{d U}\right|+\frac{|g|}{U}+|\beta g|+\frac{2 a d_{i}}{|d|^{2}}-\frac{2 d_{i}}{|d|^{2} U}+1\right\}$.

Noticing that $c>0, m_{i} d_{r}+m_{r} d_{i}>0, b>0, m_{i}>0, d_{i}>0$, and $\beta>0$, we have

$$
\frac{d}{d t}\left(\|u+g \varphi\|_{L^{2}}^{2}+\|\varphi\|_{L^{2}}^{2}\right) \leq C_{1}\left(\|u+g \varphi\|_{L^{2}}^{2}+\|\varphi\|_{L^{2}}^{2}\right)+\|f\|_{L^{2}}^{2}+\left(1+g^{2}\right)\|h\|_{L^{2}}^{2} .
$$

By Gronwall's inequality, we have

$$
\|u+g \varphi\|_{L^{2}}^{2}+\|\varphi\|_{L^{2}}^{2} \leq C .
$$

From (8), we can also deduce that

$$
\int_{0}^{T}\|u+g \varphi\|_{L^{4}}^{4} d t+\int_{0}^{T}\left\|\Lambda^{\alpha}(u+g \varphi)\right\|_{L^{2}}^{2} d t++\int_{0}^{T}\left\|\Lambda^{\alpha} \varphi\right\|_{L^{2}}^{2} d t \leq C .
$$

Thus we complete the proof.

Lemma 2 Let $u_{0} \in L^{2}(\Omega), \varphi_{0} \in L^{2}(\Omega), f \in L^{2}(\Omega), h \in L^{2}(\Omega)$. Assume that $U>0, c>0$, $m_{i} d_{r}+m_{r} d_{i}>0, b>0, m_{i}>0, d_{i}>0$ and $\beta>0$, we have

$$
(u+g \varphi)_{t} \in L^{\frac{4}{3}}\left(0, T ; H^{-\alpha}(\Omega)\right), \quad \varphi_{t} \in L^{2}\left(0, T ; H^{-\alpha}(\Omega)\right) .
$$

Proof It follows from Lemma 1 that

$$
u+g \varphi \in L^{2}\left(0, T ; H^{\alpha}(\Omega)\right), \quad \varphi \in L^{2}\left(0, T ; H^{\alpha}(\Omega)\right) .
$$

For any $\psi \in H^{\alpha}(\Omega)$, we have

$$
\begin{gathered}
\int_{0}^{t}\left((u+g \varphi)_{t}, \psi\right) d t=\frac{i a}{d} \int_{0}^{t}((u+g \varphi), \psi) d t-\frac{i}{d U} \int_{0}^{t}((u+g \varphi), \psi) d t \\
+\left(\frac{i g}{d U}-\beta g\right) \int_{0}^{t}(\varphi, \psi) d t-\frac{i c}{4 m d} \int_{0}^{t}\left(\Lambda^{2 \alpha}(u+g \varphi), \psi\right) d t \\
\quad-\frac{i b}{d} \int_{0}^{t}\left(|u+g \varphi|^{2}(u+g \varphi), \psi\right) d t+\int_{0}^{t}(f+g h, \psi) d t \\
\int_{0}^{t}\left(\varphi_{t}, \psi\right) d t=-\beta \int_{0}^{t}(\varphi, \psi) d t+\frac{i g}{U} \int_{0}^{t}(u, \psi) d t-i(2 v-2 \mu) \int_{0}^{t}(\varphi, \psi) d t \\
-\frac{i}{4 m} \int_{0}^{t}\left(\Lambda^{2 \alpha} \varphi, \psi\right) d t+\int_{0}^{t}(h, \psi) d t .
\end{gathered}
$$

By Hölder's inequality, we have

$$
\left|\int_{0}^{t}(u+g \varphi, \psi) d t\right| \leq C\|u+g \varphi\|_{L^{2}\left(0, t ; L^{2}(\Omega)\right)}\|\psi\|_{L^{2}\left(0, t ; L^{2}(\Omega)\right)},
$$




$$
\begin{aligned}
& \left|\int_{0}^{t}(\varphi, \psi) d t\right| \leq C\|\varphi\|_{L^{2}\left(0, t ; L^{2}(\Omega)\right)}\|\psi\|_{L^{2}\left(0, t ; L^{2}(\Omega)\right)} \\
& \left|\int_{0}^{t}\left(\Lambda^{2 \alpha}(u+g \varphi), \psi\right) d t\right| \leq C\|u+g \varphi\|_{L^{2}\left(0, t ; H^{\alpha}(\Omega)\right)}\|\psi\|_{L^{2}\left(0, t ; H^{\alpha}(\Omega)\right)} \\
& \left|\int_{0}^{t}\left(|u+g \varphi|^{2}(u+g \varphi), \psi\right) d t\right| \leq C\|u+g \varphi\|_{L^{4}\left(0, t ; L^{4}(\Omega)\right)}^{3}\|\psi\|_{L^{4}\left(0, t ; L^{4}(\Omega)\right)} \\
& \left|\int_{0}^{t}\left(\Lambda^{2 \alpha} \varphi, \psi\right) d t\right| \leq C\|\varphi\|_{L^{2}\left(0, t ; H^{\alpha}(\Omega)\right)}\|\psi\|_{L^{2}\left(0, t ; H^{\alpha}(\Omega)\right)} \\
& \left|\int_{0}^{t}(f+g h, \psi) d t\right| \leq C\left(\|f\|_{L^{2}\left(0, t ; L^{2}(\Omega)\right)}+\|h\|_{L^{2}\left(0, t ; L^{2}(\Omega)\right)}\right)\|\psi\|_{L^{2}\left(0, t ; L^{2}(\Omega)\right)} \\
& \left|\int_{0}^{t}(h, \psi) d t\right| \leq C\|h\|_{L^{2}\left(0, t ; L^{2}(\Omega)\right)}\|\psi\|_{L^{2}\left(0, t ; L^{2}(\Omega)\right)} .
\end{aligned}
$$

Applying the Sobolev embedding

$$
\|\psi\|_{L^{4}(\Omega)} \leq C\|\psi\|_{H^{\alpha}(\Omega)},
$$

we have

$$
\left|\int_{0}^{t}\left((u+g \varphi)_{t}, \psi\right) d t\right| \leq C\|\psi\|_{L^{4}\left(0, t ; H^{\alpha}(\Omega)\right)}, \quad\left|\int_{0}^{t}\left(\varphi_{t}, \psi\right) d t\right| \leq C\|\psi\|_{L^{2}\left(0, t ; H^{\alpha}(\Omega)\right)}
$$

Therefore

$$
\left\|(u+g \varphi)_{t}\right\|_{L^{\frac{4}{3}}\left(0, t ; H^{-\alpha}(\Omega)\right)} \leq C, \quad\left\|\varphi_{t}\right\|_{L^{2}\left(0, t ; H^{-\alpha}(\Omega)\right)} \leq C .
$$

Thus the proof of Lemma 2 is completed.

$$
\text { Let } I_{\phi}(t)=\left(\left(u+g \varphi, \phi_{1}\right),\left(\varphi, \phi_{2}\right)\right), \phi=\left(\phi_{1}, \phi_{2}\right) \text {. }
$$

Lemma 3 Under the conditions as in Lemma 1 , for any $\phi_{1} \in L^{2}(\Omega), \phi_{2} \in L^{2}(\Omega), I_{\phi}(t)$ is a continuous function with respect to $t$.

Proof First, let $\phi=\left(\phi_{1}, \phi_{2}\right) \in C^{\infty}(\Omega) \times C^{\infty}(\Omega)$. We have

$$
\begin{aligned}
I_{\phi_{1}}(t)= & \left(u+g \varphi, \phi_{1}\right)=\left(u_{0}+g \varphi_{0}, \phi_{1}\right)+\frac{i a}{d} \int_{0}^{t}\left((u+g \varphi), \phi_{1}\right) d t \\
& -\frac{i}{d U} \int_{0}^{t}\left((u+g \varphi), \phi_{1}\right) d t+\left(\frac{i g}{d U}-\beta g\right) \int_{0}^{t}\left(\varphi, \phi_{1}\right) d t \\
& -\frac{i c}{4 m d} \int_{0}^{t}\left(\Lambda^{2 \alpha}(u+g \varphi), \phi_{1}\right) d t-\frac{i b}{d} \int_{0}^{t}\left(|u+g \varphi|^{2}(u+g \varphi), \phi_{1}\right) d t \\
& +\int_{0}^{t}\left(f+g h, \phi_{1}\right) d t, \\
I_{\phi_{2}}(t)= & \left(\varphi, \phi_{2}\right)=\left(\varphi_{0}, \phi_{2}\right)-\beta \int_{0}^{t}\left(\varphi, \phi_{2}\right) d t+\frac{i g}{U} \int_{0}^{t}\left(u, \phi_{2}\right) d t \\
& -i(2 v-2 \mu) \int_{0}^{t}\left(\varphi, \phi_{2}\right) d t-\frac{i}{4 m} \int_{0}^{t}\left(\Lambda^{2 \alpha} \varphi, \phi_{2}\right) d t+\int_{0}^{t}\left(h, \phi_{2}\right) d t .
\end{aligned}
$$


For $0 \leq t_{1}, t_{2} \leq T$ and $\left|t_{2}-t_{1}\right|<1$, we have

$$
\begin{aligned}
\left|I_{\phi_{1}}\left(t_{1}\right)-I_{\phi_{1}}\left(t_{2}\right)\right| \leq & C\left(\left\|\phi_{1}\right\|_{L^{\infty}}+\left\|\Lambda^{2 \alpha} \phi_{1}\right\|_{L^{\infty}}\right)\|u+g \varphi\|_{L^{2}\left(t_{1}, t_{2} ; L^{2}\right)}\left|t_{1}-t_{2}\right|^{\frac{1}{2}} \\
& +C\left\|\phi_{1}\right\|_{L^{\infty}}\|\varphi\|_{L^{2}\left(t_{1}, t_{2} ; L^{2}\right)}\left|t_{1}-t_{2}\right|^{\frac{1}{2}} \\
& +C\left\|\phi_{1}\right\|_{L^{\infty}}\|u+g \varphi\|_{L^{4}\left(t_{1}, t_{2} ; L^{4}\right)}^{3}\left|t_{1}-t_{2}\right|^{\frac{1}{4}} \\
& +C\left\|\phi_{1}\right\|_{L^{\infty}}\left(\|f\|_{L^{2}\left(t_{1}, t_{2} ; L^{2}\right)}+\|h\|_{L^{2}\left(t_{1}, t_{2} ; L^{2}\right)}\right)\left|t_{1}-t_{2}\right|^{\frac{1}{2}} \\
\leq & C\left|t_{1}-t_{2}\right|^{\frac{1}{4}} \\
\left|I_{\phi_{2}}\left(t_{1}\right)-I_{\phi_{2}}\left(t_{2}\right)\right| \leq & C\left(\left\|\phi_{2}\right\|_{L^{\infty}}+\left\|\Lambda^{2 \alpha} \phi_{2}\right\|_{L^{\infty}}\right)\|\varphi\|_{L^{2}\left(t_{1}, t_{2} ; L^{2}\right)}\left|t_{1}-t_{2}\right|^{\frac{1}{2}} \\
& +C\left\|\phi_{2}\right\|_{L^{\infty}}\|u+g \varphi\|_{L^{2}\left(t_{1}, t_{2} ; L^{2}\right)}\left|t_{1}-t_{2}\right|^{\frac{1}{2}} \\
& +C\left\|\phi_{2}\right\|_{L^{\infty}}\|h\|_{L^{2}\left(t_{1}, t_{2} ; L^{2}\right)}\left|t_{1}-t_{2}\right|^{\frac{1}{2}} \\
\leq & C\left|t_{1}-t_{2}\right|^{\frac{1}{2}} \leq C\left|t_{1}-t_{2}\right|^{\frac{1}{4}}
\end{aligned}
$$

Then

$$
\left|I_{\phi}\left(t_{1}\right)-I_{\phi}\left(t_{2}\right)\right| \leq\left|I_{\phi_{1}}\left(t_{1}\right)-I_{\phi_{1}}\left(t_{2}\right)\right|+\left|I_{\phi_{2}}\left(t_{1}\right)-I_{\phi_{2}}\left(t_{2}\right)\right| \leq C\left|t_{1}-t_{2}\right|^{\frac{1}{4}}
$$

Therefore, the continuity of $I_{\phi}(t)$ follows.

Next we use a density argument to extend the result for $\phi \in L^{2}(\Omega) \times L^{2}(\Omega)$. Let $\epsilon>0$ be an arbitrary positive number, for $\phi \in L^{2}(\Omega) \times L^{2}(\Omega)$, we may select some $\phi^{\epsilon} \in C^{\infty}(\Omega) \times$ $C^{\infty}(\Omega)$ such that $\left\|\phi^{\epsilon}-\phi\right\|_{L^{2}(\Omega)} \leq \epsilon$. By the triangle inequality and Hölder's inequality, we have

$$
\begin{aligned}
\left|I_{\phi}\left(t_{1}\right)-I_{\phi}\left(t_{2}\right)\right| \leq & \left|I_{\phi_{1}}\left(t_{1}\right)-I_{\phi_{1}^{\epsilon}}\left(t_{1}\right)\right|+\left|I_{\phi_{1}^{\epsilon}}\left(t_{1}\right)-I_{\phi_{1}^{\epsilon}}\left(t_{2}\right)\right| \\
& +\left|I_{\phi_{1}}\left(t_{2}\right)-I_{\phi_{1}^{\epsilon}}\left(t_{2}\right)\right|+\left|I_{\phi_{2}}\left(t_{1}\right)-I_{\phi_{2}^{\epsilon}}\left(t_{1}\right)\right| \\
& +\left|I_{\phi_{2}^{\epsilon}}\left(t_{1}\right)-I_{\phi_{2}^{\epsilon}}\left(t_{2}\right)\right|+\left|I_{\phi_{2}}\left(t_{2}\right)-I_{\phi_{2}^{\epsilon}}\left(t_{2}\right)\right| \\
\leq & \epsilon C+\left|I_{\phi^{\epsilon}}\left(t_{1}\right)-I_{\phi^{\epsilon}}\left(t_{2}\right)\right| .
\end{aligned}
$$

Since $I_{\phi^{\epsilon}}$ is continuous in $t$ and $\epsilon$ is arbitrary, the continuity of $I_{\phi}(t)$ follows for $\phi \in L^{2} \times$ $L^{2}$.

\section{Global existence of weak solutions}

In this section, we will show the global existence of the weak solutions. We first give the definition of weak solutions.

Definition Let $u_{0} \in L^{2}(\Omega), \varphi_{0} \in L^{2}(\Omega), f(x) \in L^{2}(\Omega), h(x) \in L^{2}(\Omega)$, we say that $(u, \varphi)$ is a weak solution of (1)-(4) if

(i) for all $T>0, u \in L^{\infty}\left(0, T ; L^{2}(\Omega)\right) \cap L^{2}\left(0, T ; H^{\alpha}(\Omega)\right), \varphi \in L^{\infty}\left(0, T ; L^{2}(\Omega)\right) \cap L^{2}(0, T$; $\left.H^{\alpha}(\Omega)\right)$; 
(ii) for all $\psi \in C^{\infty}\left(Q_{T}\right)$, we have

$$
\begin{gathered}
-i d(u, \psi)+i d\left(u_{0}, \psi\right) \\
=\left(-\frac{d g^{2}+1}{U}+a\right) \int_{0}^{t}(u, \psi) d t+g[a+d(2 v-2 \mu)] \int_{0}^{t}(\varphi, \psi) d t \\
-\frac{c}{4 m} \int_{0}^{t}\left(\Lambda^{\alpha} u, \Lambda^{\alpha} \psi\right) d t-\frac{g}{4 m}(c-d) \int_{0}^{t}\left(\Lambda^{\alpha} \varphi, \Lambda^{\alpha} \psi\right) d t \\
-b \int_{0}^{t}\left(|u+g \varphi|^{2}(u+g \varphi), \psi\right) d t+i d \int_{0}^{t}(f(x), \psi) d t, \\
i(\varphi, \psi)-i\left(\varphi_{0}, \psi\right)=-i \beta \int_{0}^{t}(\varphi, \psi) d t-\frac{g}{U} \int_{0}^{t}(u, \psi) d t+(2 v-2 \mu) \int_{0}^{t}(\varphi, \psi) d t \\
\quad+\frac{1}{4 m} \int_{0}^{t}\left(\Lambda^{\alpha} \varphi, \Lambda^{\alpha} \psi\right) d t+i \int_{0}^{t}(h(x), \psi) d t,
\end{gathered}
$$

with initial conditions

$$
(u(x, 0), \psi)=\left(u_{0}(x), \psi\right), \quad(\varphi(x, 0), \psi)=\left(\varphi_{0}(x), \psi\right),
$$

where $Q_{T}=(0, T) \times \Omega$.

Next, we recall the following lemmas which will be used later.

Lemma 4 Let $B_{0}, B, B_{1}$ be three Banach spaces such that

$$
B_{0} \subset B \subset B_{1},
$$

where the injections are continuous and $B_{0}, B_{1}$ are reflexive, and the injection $B_{0} \rightarrow B$ is compact. Denote

$$
W=\left\{v \mid v \in L^{p_{0}}\left(0, T ; B_{0}\right), v^{\prime}=\frac{d v}{d t} \in L^{p_{1}}\left(0, T ; B_{1}\right)\right\}
$$

where $T$ is finite and $1<p_{0}, p_{1}<\infty$. Then $W$ equipped with the norm

$$
\|v\|_{L^{p_{0}\left(0, T ; B_{0}\right)}}+\left\|v^{\prime}\right\|_{L^{p_{1}\left(0, T ; B_{1}\right)}}
$$

is a Banach space and the embedding $W \hookrightarrow L^{p_{0}}(0, T ; B)$ is compact.

Lemma 5 Assume that $D$ is a bounded domain in $\mathbb{R}_{x}^{n} \times \mathbb{R}_{t}$, functions $f_{l}, f \in L^{q}(D)(1<q<$ $\infty)$ and

$$
\left\|f_{l}\right\|_{L^{q}(D)} \leq C, \quad f_{l} \rightarrow f \text { in } D \text { a.e. }
$$

Then $f_{l} \rightarrow f$ weakly in $L^{q}(D)$.

Lemma $6 X$ is a Banach space, suppose that $g \in L^{p}(0, T ; X), \frac{\partial g}{\partial t} \in L^{p}(0, T ; X)(1 \leq p \leq \infty)$. Then $g \in C([0, T] ; X)$ (after possibly being redefined on a set of measure zero). 
Now, we state our main result as follows.

Theorem 1 Let $\frac{1}{2}<\alpha \leq 1, u_{0} \in L^{2}(\Omega), \varphi_{0} \in L^{2}(\Omega), f(x) \in L^{2}(\Omega), h(x) \in L^{2}(\Omega), U>0, c>$ $0, m_{i} d_{r}+m_{r} d_{i}>0, b>0, m_{i}>0, d_{i}>0$, and $\beta>0$, then there exists a unique weak solution $(u, \varphi)$ to (1)-(4) such that

$$
\begin{aligned}
& u+g \varphi \in C\left([0, T] ; w-L^{2}(\Omega)\right) \cap L^{2}\left(0, T ; H^{\alpha}(\Omega)\right), \\
& \varphi \in C\left([0, T] ; w-L^{2}(\Omega)\right) \cap L^{2}\left(0, T ; H^{\alpha}(\Omega)\right),
\end{aligned}
$$

and

$$
u+g \varphi \in L^{4}\left(0, T ; L^{4}(\Omega)\right)
$$

where we say that $u+g \varphi \in C\left([0, T] ; w-L^{2}(\Omega)\right)$ and $\varphi \in C\left([0, T] ; w-L^{2}(\Omega)\right)$, if $\left(u+g \varphi, \phi_{1}\right) \in$ $C([0, T])$ and $\left(\varphi, \phi_{2}\right) \in C([0, T])$ for any $\phi=\left(\phi_{1}, \phi_{2}\right) \in L^{2} \times L^{2}$.

Proof By Galerkin's method, we look for approximate solutions $\left(u_{N}(x, t), \varphi_{N}(x, t)\right)$ for equations (1)-(4) in the form

$$
u_{N}(x, t)=\sum_{j=1}^{N} \alpha_{j N}(t) \omega_{j}(x), \quad \varphi_{N}(x, t)=\sum_{j=1}^{N} \beta_{j N}(t) \omega_{j}(x)
$$

where $\omega_{j}(x)=e^{i j \cdot x}$ and $\alpha_{j N}(t), \beta_{j N}(t)$ satisfy the following system of ordinary differential equations:

$$
\begin{aligned}
-i d\left(u_{N t}, \omega_{j}\right)= & \left(-\frac{d g^{2}+1}{U}+a\right)\left(u_{N}, \omega_{j}\right)+g[a+d(2 v-2 \mu)]\left(\varphi_{N}, \omega_{j}\right) \\
& -\frac{c}{4 m}\left(\Lambda^{2 \alpha} u_{N}, \omega_{j}\right)-\frac{g}{4 m}(c-d)\left(\Lambda^{2 \alpha} \varphi_{N}, \omega_{j}\right) \\
& -b\left(\left|u_{N}+g \varphi_{N}\right|^{2}\left(u_{N}+g \varphi_{N}\right), \omega_{j}\right)+i d\left(f(x), \omega_{j}\right), \\
i\left(\varphi_{N t}, \omega_{j}\right)=-i \beta\left(\varphi_{N}, \omega_{j}\right)-\frac{g}{U}\left(u_{N}, \omega_{j}\right)+(2 v-2 \mu)\left(\varphi_{N}, \omega_{j}\right) & \\
+ & \frac{1}{4 m}\left(\Lambda^{2 \alpha} \varphi_{N}, \omega_{j}\right)+i\left(h(x), \omega_{j}\right), \quad 1 \leq j \leq N, \\
u_{N}(x, 0)= & u_{N 0}(x) \rightarrow u_{0}(x), \quad \varphi_{N}(x, 0)=\varphi_{N 0}(x) \rightarrow \varphi_{0}(x) \quad \text { in } L^{2}(\Omega) .
\end{aligned}
$$

The local existence theory for nonlinear ordinary differential equations ensures that the initial value problem (9)-(11) has at least one solution on $\left[0, t_{m}\right]$. By a priori estimates, we know that there exists a global solution for the initial value problem of the nonlinear ordinary differential system (9)-(11) on $[0, T]$.

Similar to the proof of Lemma 1 and Lemma 2, we have

$$
\begin{aligned}
& u_{N}+g \varphi_{N} \in L^{2}\left(0, T ; H^{\alpha}(\Omega)\right), \quad u_{N t}+g \varphi_{N t} \in L^{\frac{4}{3}}\left(0, T ; H^{-\alpha}(\Omega)\right), \\
& u_{N}+g \varphi_{N} \in L^{4}\left(0, T ; L^{4}(\Omega)\right), \\
& \varphi_{N} \in L^{2}\left(0, T ; H^{\alpha}(\Omega)\right), \quad \varphi_{N t} \in L^{2}\left(0, T ; H^{-\alpha}(\Omega)\right) .
\end{aligned}
$$


Applying the compactness lemma (Lemma 4), there exist two subsequences, still denoted by $\left\{u_{N}+g \varphi_{N}\right\},\left\{\varphi_{N}\right\}$, such that

$$
\begin{aligned}
& u_{N}+g \varphi_{N} \rightarrow u+g \varphi \quad \text { strongly in } L^{2}\left(0, T ; L^{2}(\Omega)\right) \text { and a.e. } \\
& \varphi_{N} \rightarrow \varphi \text { strongly in } L^{2}\left(0, T ; L^{2}(\Omega)\right) \text { and a.e. }
\end{aligned}
$$

Since $u_{N}+g \varphi_{N} \in L^{4}\left(0, T ; L^{4}(\Omega)\right)$, we can deduce that

$$
\left\|\left|u_{N}+g \varphi_{N}\right|^{2}\left(u_{N}+g \varphi_{N}\right)\right\|_{L^{\frac{4}{3}}\left(0, T ; L^{\frac{4}{3}}(\Omega)\right)} \leq C .
$$

From Lemma 5, we have

$$
\left|u_{N}+g \varphi_{N}\right|^{2}\left(u_{N}+g \varphi_{N}\right) \rightarrow|u+g \varphi|^{2}(u+g \varphi) \quad \text { weakly in } L^{\frac{4}{3}}\left(0, T ; L^{\frac{4}{3}}(\Omega)\right) .
$$

For any $\phi \in L^{2} \times L^{2}$, we see that $\left\{\left(u+g \varphi, \phi_{1}\right)\right\}_{N}$ and $\left\{\left(\varphi, \phi_{2}\right)\right\}_{N}$ are equicontinuous in $C([0, T])$ from Lemma 3. On the other hand, it follows from Lemma 1 that $\left\{\left(u+g \varphi, \phi_{1}\right)\right\}_{N}$ and $\left\{\left(\varphi, \phi_{2}\right)\right\}_{N}$ are uniformly bounded in $C([0, T])$. Therefore, by Arzela-Ascoli theorem, we have $\left\{\left(u+g \varphi, \phi_{1}\right)\right\}_{N}$ and $\left\{\left(\varphi, \phi_{2}\right)\right\}_{N}$ are compact in $C([0, T])$.

Letting $N \rightarrow \infty$, we immediately obtain

$$
\begin{aligned}
& \int_{0}^{t}\left(u_{N}+g \varphi_{N}, \psi\right) d t \rightarrow \int_{0}^{t}(u+g \varphi, \psi) d t, \\
& \int_{0}^{t}\left(\Lambda^{\alpha}\left(u_{N}+g \varphi_{N}\right), \Lambda^{\alpha} \psi\right) d t \rightarrow \int_{0}^{t}\left(\Lambda^{\alpha}(u+g \varphi), \Lambda^{\alpha} \psi\right) d t, \\
& \int_{0}^{t}\left(\left|u_{N}+g \varphi_{N}\right|^{2}\left(u_{N}+g \varphi_{N}\right), \psi\right) d t \rightarrow \int_{0}^{t}\left(|u+g \varphi|^{2}(u+g \varphi), \psi\right) d t, \\
& \int_{0}^{t}\left(\varphi_{N}, \psi\right) d t \rightarrow \int_{0}^{t}(\varphi, \psi) d t, \\
& \int_{0}^{t}\left(\Lambda^{\alpha} \varphi_{N}, \Lambda^{\alpha} \psi\right) d t \rightarrow \int_{0}^{t}\left(\Lambda^{\alpha} \varphi, \Lambda^{\alpha} \psi\right) d t .
\end{aligned}
$$

Therefore, we have for any $\psi \in C^{\infty}\left(Q_{T}\right)$

$$
\begin{aligned}
-i d(u, \psi)+i d\left(u_{0}, \psi\right)= & \left(-\frac{d g^{2}+1}{U}+a\right) \int_{0}^{t}(u, \psi) d t+g[a+d(2 v-2 \mu)] \int_{0}^{t}(\varphi, \psi) d t \\
& -\frac{c}{4 m} \int_{0}^{t}\left(\Lambda^{\alpha} u, \Lambda^{\alpha} \psi\right) d t-\frac{g}{4 m}(c-d) \int_{0}^{t}\left(\Lambda^{\alpha} \varphi, \Lambda^{\alpha} \psi\right) d t \\
& -b \int_{0}^{t}\left(|u+g \varphi|^{2}(u+g \varphi), \psi\right) d t-i d \int_{0}^{t}(f, \psi) d t, \\
i(\varphi, \psi)-i\left(\varphi_{0}, \psi\right)=-i \beta \int_{0}^{t}(\varphi, \psi) d t-\frac{g}{U} \int_{0}^{t}(u, \psi) d t+(2 v-2 \mu) \int_{0}^{t}(\varphi, \psi) d t & +\frac{1}{4 m} \int_{0}^{t}\left(\Lambda^{\alpha} \varphi, \Lambda^{\alpha} \psi\right) d t+i \int_{0}^{t}(h, \psi) d t .
\end{aligned}
$$

Next we show the initial conditions hold. 
By $u_{N}+g \varphi_{N} \in L^{2}\left(0, T ; H^{\alpha}(\Omega)\right), u_{N t}+g \varphi_{N t} \in L^{2}\left(0, T ; H^{-\alpha}(\Omega)\right)$, and Lemma 6 , we have

$$
u_{N}+g \varphi_{N} \in C\left([0, T], H^{-\alpha}(\Omega)\right)
$$

Similarly, $\varphi_{N} \in C\left([0, T], H^{-\alpha}(\Omega)\right)$. Then

$$
\begin{aligned}
& u_{N}(0)+g \varphi_{N}(0) \rightarrow u(0)+g \varphi(0) \quad \text { weakly in } H^{-\alpha}(\Omega), \\
& \varphi_{N}(0) \rightarrow \varphi(0) \quad \text { weakly in } H^{-\alpha}(\Omega) .
\end{aligned}
$$

But from (12), we have

$$
\begin{aligned}
& u_{N}(0)+g \varphi_{N}(0) \rightarrow u_{0}+g \varphi_{0} \quad \text { in } L^{2}(\Omega), \\
& \varphi_{N}(0) \rightarrow \varphi_{0} \quad \text { in } L^{2}(\Omega) .
\end{aligned}
$$

Therefore

$$
u(0)+g \varphi(0)=u_{0}+g \varphi_{0}, \quad \varphi(0)=\varphi_{0} .
$$

Assume that $(u, \varphi)$ and $\left(u^{\star}, \varphi^{\star}\right)$ are the two different solutions of (1)-(4). For $w=u-u^{\star}, v=$ $\varphi-\varphi^{\star}$, we have

$$
\begin{aligned}
& (w+g v)_{t}=-\frac{i}{d U}(w+g v)+\frac{i g}{d U} v+\frac{i a}{d}(w+g v)-\frac{i c}{4 m d} \Lambda^{2 \alpha}(w+g v) \\
& \quad-\frac{i b}{d}\left(|u+g \varphi|^{2}(u+g \varphi)-\left|u^{\star}+g \varphi^{\star}\right|^{2}\left(u^{\star}+g \varphi^{\star}\right)\right), \\
& v_{t}=-\beta v+\frac{i g}{U} w-i(2 v-2 \mu) v-\frac{i}{4 m} \Lambda^{2 \alpha} v, \\
& w(x, 0)=0, \quad v(x, 0)=0 .
\end{aligned}
$$

Multiplying (12)-(13) by $\overline{(w+g v)}$ and $\bar{v}$, respectively, then integrating over $\Omega$ and taking the real part, we have

$$
\begin{aligned}
& \frac{1}{2} \frac{d}{d t}\|w+g v\|_{L^{2}}^{2} \\
& =\left(\frac{a d_{i}}{|d|^{2}}-\frac{d_{i}}{|d|^{2} U}\right)\|w+g v\|_{L^{2}}^{2}+\operatorname{Re}\left(\frac{i g}{d U} \int_{\Omega} v \overline{(w+g v)} d x\right) \\
& \quad-\frac{c\left(m_{i} d_{r}+m_{r} d_{i}\right)}{4|m|^{2}|d|^{2}}\left\|\Lambda^{\alpha}(u+g \varphi)\right\|_{L^{2}}^{2} \\
& \quad-\operatorname{Re}\left(\frac{i b}{d} \int_{\Omega}\left(|u+g \varphi|^{2}(u+g \varphi)-\left|u^{\star}+g \varphi^{\star}\right|^{2}\left(u^{\star}+g \varphi^{\star}\right)\right) \overline{(w+g v)} d x\right), \\
& \frac{1}{2} \frac{d}{d t}\|v\|_{L^{2}}^{2}=-\beta\|v\|_{L^{2}}^{2}+\operatorname{Re}\left(\frac{i g}{U} \int_{\Omega} w \bar{v} d x\right)-\frac{m_{i}}{4|m|^{2}}\left\|\Lambda^{\alpha} \varphi\right\|_{L^{2}}^{2} .
\end{aligned}
$$

Combining the above two equations yields 


$$
\begin{aligned}
& \frac{1}{2} \frac{d}{d t}\left(\|w+g v\|_{L^{2}}^{2}+\|v\|_{L^{2}}^{2}\right) \\
& \leq\left(\frac{a d_{i}}{|d|^{2}}-\frac{d_{i}}{|d|^{2} U}+\frac{g}{2|d| U}+\frac{g}{2 U}\right)\|w+g v\|_{L^{2}}^{2}-\frac{c\left(m_{i} d_{r}+m_{r} d_{i}\right)}{4|m|^{2}|d|^{2}}\left\|\Lambda^{\alpha}(w+g v)\right\|_{L^{2}}^{2} \\
& +\frac{3 b}{|d|} \int_{\Omega} \max \left(|u+g \varphi|^{2},\left|u^{\star}+g \varphi^{\star}\right|^{2}\right)|w+g v|^{2} d x+\left(\frac{g}{2|d| U}+\frac{g}{2 U}-\beta\right)\|v\|_{L^{2}}^{2} \\
& \leq\left(\frac{a d_{i}}{|d|^{2}}-\frac{d_{i}}{|d|^{2} U}+\frac{g}{2|d| U}+\frac{g}{2 U}\right)\|w+g v\|_{L^{2}}^{2}-\frac{c\left(m_{i} d_{r}+m_{r} d_{i}\right)}{4|m|^{2}|d|^{2}}\left\|\Lambda^{\alpha}(w+g v)\right\|_{L^{2}}^{2} \\
& +\frac{3 b}{|d|} \int_{\Omega} \max \left(|u+g \varphi|^{2},\left|u^{\star}+g \varphi^{\star}\right|^{2}\right)|w+g v|^{2} d x+\left(\frac{g}{2|d| U}+\frac{g}{2 U}-\beta\right)\|v\|_{L^{2}}^{2} \\
& \leq\left(\frac{a d_{i}}{|d|^{2}}-\frac{d_{i}}{|d|^{2} U}+\frac{g}{2|d| U}+\frac{g}{2 U}\right)\|w+g v\|_{L^{2}}^{2}-\frac{c\left(m_{i} d_{r}+m_{r} d_{i}\right)}{4|m|^{2}|d|^{2}}\left\|\Lambda^{\alpha}(w+g v)\right\|_{L^{2}}^{2} \\
& +\frac{3 b}{|d|}\left(\int_{\Omega}|u+g \varphi|^{4} d x\right)^{\frac{1}{2}}\left(\int_{\Omega}|w+g v|^{4} d x\right)^{\frac{1}{2}} \\
& +\frac{3 b}{|d|}\left(\int_{\Omega}\left|u^{\star}+g \varphi^{\star}\right|^{4} d x\right)^{\frac{1}{2}}\left(\int_{\Omega}|w+g \nu|^{4} d x\right)^{\frac{1}{2}}+\left(\frac{g}{2|d| U}+\frac{g}{2 U}-\beta\right)\|v\|_{L^{2}}^{2} \\
& \leq\left(\frac{a d_{i}}{|d|^{2}}-\frac{d_{i}}{|d|^{2} U}+\frac{g}{2|d| U}+\frac{g}{2 U}\right)\|w+g v\|_{L^{2}}^{2}-\frac{c\left(m_{i} d_{r}+m_{r} d_{i}\right)}{4|m|^{2}|d|^{2}}\left\|\Lambda^{\alpha}(w+g v)\right\|_{L^{2}}^{2} \\
& +\frac{3 b}{|d|}\left(\|u+g \varphi\|_{L^{4}}^{2}+\left\|u^{\star}+g \varphi^{\star}\right\|_{L^{4}}^{2}\right)\|w+g v\|_{L^{2}}^{2\left(1-\frac{1}{4 \alpha}\right)}\left\|\Lambda^{\alpha}(w+g v)\right\|_{L^{2}}^{\frac{1}{2 \alpha}} \\
& +\left(\frac{g}{2|d| U}+\frac{g}{2 U}-\beta\right)\|v\|_{L^{2}}^{2} \\
& \leq\left(\frac{a d_{i}}{|d|^{2}}-\frac{d_{i}}{|d|^{2} U}+\frac{g}{2|d| U}+\frac{g}{2 U}\right)\|w+g v\|_{L^{2}}^{2}-\frac{c\left(m_{i} d_{r}+m_{r} d_{i}\right)}{4|m|^{2}|d|^{2}}\left\|\Lambda^{\alpha}(w+g v)\right\|_{L^{2}}^{2} \\
& +\frac{c\left(m_{i} d_{r}+m_{r} d_{i}\right)}{4|m|^{2}|d|^{2}}\left\|\Lambda^{\alpha}(w+g v)\right\|_{L^{2}}^{2}+C\left(\|u+g \varphi\|_{L^{4}}^{\frac{8 \alpha}{4 \alpha-1}}+\left\|u^{\star}+g \varphi^{\star}\right\|_{L^{4}}^{\frac{8 \alpha}{4 \alpha-1}}\right)\|w+g v\|_{L^{2}}^{2} \\
& +\left(\frac{g}{2|d| U}+\frac{g}{2 U}-\beta\right)\|v\|_{L^{2}}^{2} \\
& \leq C\left(\|u+g \varphi\|_{L^{4}}^{\frac{8 \alpha}{4 \alpha-1}}+\left\|u^{\star}+g \varphi^{\star}\right\|_{L^{4}}^{\frac{8 \alpha}{4 \alpha-1}}+1\right)\left(\|w+g v\|_{L^{2}}^{2}+\|v\|_{L^{2}}^{2}\right),
\end{aligned}
$$

where we have used the Gagliardo-Nirenberg inequality, the Young inequality, and the elementary inequality

$$
|a|^{p} a-|b|^{p} b \leq(p+1) \max \left(|a|^{p},|b|^{p}\right)|a-b| .
$$

Note that $w(x, 0)=0$ and $v(x, 0)=0$. By Gronwall's inequality, we have

$$
w+g v=0, \quad v=0 .
$$

Thus we complete the proof.

\section{Global attractors}

Finally, we consider the large time behavior of the solution to (1)-(4) in $L^{2} \times L^{2}$. Before doing so, we give the following lemma which will be used later. 
Lemma 7 ([16]) Let $g, h$ and $y$ be three nonnegative locally integrable functions on $\left(t_{0}, \infty\right)$ such that $y^{\prime}$ is locally integrable on $\left(t_{0}, \infty\right)$, and which satisfy

$$
\frac{d y}{d t} \leq g y+h
$$

and

$$
\int_{t}^{t+r} g(s) d s \leq a_{1}, \quad \int_{t}^{t+r} h(s) d s \leq a_{2}, \quad \int_{t}^{t+r} y(s) d s \leq a_{3}, \quad t \geq t_{0},
$$

where $r, a_{1}, a_{2}, a_{3}$ are positive constants. Then

$$
y(t+r) \leq\left(\frac{a_{3}}{r}+a_{2}\right) e^{a_{1}}, \quad t \geq t_{0}
$$

Theorem 2 Assume that $\alpha \in\left(\frac{1}{2}, 1\right], U>0, c>0, m_{i} d_{r}+m_{r} d_{i}>0, b>0, m_{i}>0, d_{i}>0$, $0<g<2$ and $\beta>\frac{\frac{g}{|d| u}+\frac{g}{u}+1}{2-g}$, then the solution operator $S(t): S(t)\left(u_{0}+g \varphi_{0}, \varphi_{0}\right)=(u+g \varphi, \varphi)$, for all $t>0$, well defines a semigroup in the space $L^{2} \times L^{2}$ and the following statements hold:

(i) For any $t>0, S(t)$ is continuous in $L^{2} \times L^{2}$.

(ii) For any $\left(u_{0}, \varphi_{0}\right) \in L^{2} \times L^{2}, S(t)$ is continuous from $[0, T]$ to $L^{2} \times L^{2}$.

(iii) For any $t>0, S(t)$ is compact in $L^{2} \times L^{2}$.

(iv) The semigroup $\{S(t)\}_{t \geq 0}$ possesses a global attractor $\mathcal{A}$ in $L^{2} \times L^{2}$.

Proof It is easy to show that the solution $(u, \varphi)$ to (1)-(4) well defines a semigroup $S(t)$ on $L^{2} \times L^{2}$.

First, we consider the absorbing set in $L^{2} \times L^{2}$. Similarly to the proof of Lemma 1, we have

$$
\begin{aligned}
& \frac{d}{d t}\left(\|u+g \varphi\|_{L^{2}}^{2}+\|\varphi\|_{L^{2}}^{2}\right)-\left(\frac{g}{|d| U}+\frac{g}{U}+\frac{2 a d_{i}}{|d|^{2}}-\frac{2 d_{i}}{|d|^{2} U}+\beta g+1\right)\|u+g \varphi\|_{L^{2}}^{2} \\
& \quad+\left(2 \beta-\frac{g}{|d| U}-\frac{g}{U}-\beta g-1\right)\|\varphi\|_{L^{2}}^{2}+\frac{2 b d_{i}}{|d|^{2}}\|u+g \varphi\|_{L^{4}}^{4}+\frac{m_{i}}{4|m|^{2}}\left\|\Lambda^{\alpha} \varphi\right\|_{L^{2}}^{2} \\
& \quad+\frac{c\left(m_{i} d_{r}+m_{r} d_{i}\right)}{4|m|^{2}|d|^{2}}\left\|\Lambda^{\alpha}(u+g \varphi)\right\|_{L^{2}}^{2} d s \\
& \leq\|f\|_{L^{2}}^{2}+\left(1+g^{2}\right)\|h\|_{L^{2}}^{2} .
\end{aligned}
$$

Since

$$
\frac{2 b d_{i}}{|d|^{2} \epsilon}\|u+g \varphi\|_{L^{2}}^{2} \leq \frac{b d_{i}}{|d|^{2}}\|u+g \varphi\|_{L^{4}}^{4}+\frac{b d_{i}}{|d|^{2} \epsilon^{2}}|\Omega| .
$$

Thus we can choose $\epsilon_{0}$ small enough such that

$$
\frac{4 b d_{i}}{|d|^{2} \epsilon_{0}}-\frac{g}{|d| U}-\frac{g}{U}-\frac{2 a d_{i}}{|d|^{2}}+\frac{2 d_{i}}{|d|^{2} U}-\beta g-1>0
$$

Define

$$
R_{1}=\min \left\{\frac{4 b d_{i}}{|d|^{2} \epsilon_{0}}-\frac{g}{|d| U}-\frac{g}{U}-\frac{2 a d_{i}}{|d|^{2}}+\frac{2 d_{i}}{|d|^{2} U}-\beta g-1,2 \beta-\frac{g}{|d| U}-\frac{g}{U}-\beta g-1\right\}>0,
$$


we have

$$
\frac{d}{d t}\left(\|u+g \varphi\|_{L^{2}}^{2}+\|\varphi\|_{L^{2}}^{2}\right)+R_{1}\left(\|u+g \varphi\|_{L^{2}}^{2}+\|\varphi\|_{L^{2}}^{2}\right) \leq \frac{2 b d_{i}}{|d|^{2} \epsilon_{0}^{2}}|\Omega|+\|f\|_{L^{2}}^{2}+\left(1+g^{2}\right)\|h\|_{L^{2}}^{2} .
$$

Applying Gronwall's inequality yields

$$
\begin{aligned}
\|u+g \varphi\|_{L^{2}}^{2}+\|\varphi\|_{L^{2}}^{2} \leq & e^{-R_{1} t}\left(\left\|u_{0}+g \varphi_{0}\right\|_{L^{2}}^{2}+\left\|\varphi_{0}\right\|_{L^{2}}^{2}\right) \\
& +\left(\frac{2 b d_{i}}{|d|^{2} \epsilon_{0}^{2}}|\Omega|+\|f\|_{L^{2}}^{2}+\left(1+g^{2}\right)\|h\|_{L^{2}}^{2}\right)\left(1-e^{-R_{1} t}\right), \quad t \geq 0 .
\end{aligned}
$$

Therefore

$$
\lim _{t \rightarrow \infty}\left(\|u+g \varphi\|_{L^{2}}^{2}+\|\varphi\|_{L^{2}}^{2}\right) \leq \rho_{0}^{2}
$$

where $\rho_{0}^{2}=\frac{2 b d_{i}}{|d|^{2} \epsilon_{0}^{2}}|\Omega|+\|f\|_{L^{2}}^{2}+\left(1+g^{2}\right)\|h\|_{L^{2}}^{2}$.

It follows that the existence of an absorbing ball in $L^{2} \times L^{2}$. Indeed, for any $\rho>\rho_{0}$, denote by $\mathcal{B}_{0}$ the ball $B(0, \rho)$, for any bounded set $\mathcal{B}$, there exists a positive $t_{0}=\frac{1}{R_{1}} \log \frac{\rho^{2}}{\rho^{2}-\rho_{0}^{2}}$ such that $S(t) \mathcal{B} \subset \mathcal{B}_{0}$ for all $t \geq t_{0}$.

Next we consider the absorbing set in $H^{\alpha} \times H^{\alpha}$.

Integrating (15) from $t$ to $t+1$, and using the definition of $R_{1}$, we have

$$
\begin{gathered}
\|u(t+1)+g \varphi(t+1)\|_{L^{2}}^{2}+\|\varphi(t+1)\|_{L^{2}}^{2}+R_{1} \int_{t}^{t+1}\left(\|u+g \varphi\|_{L^{2}}^{2}+\|\varphi\|_{L^{2}}^{2}\right) d s \\
\quad+\frac{c\left(m_{i} d_{r}+m_{r} d_{i}\right)}{4|m|^{2}|d|^{2}} \int_{t}^{t+1}\left\|\Lambda^{\alpha}(u+g \varphi)\right\|_{L^{2}}^{2} d s+\frac{m_{i}}{4|m|^{2}} \int_{t}^{t+1}\left\|\Lambda^{\alpha} \varphi\right\|_{L^{2}}^{2} d s \\
\leq\|u(t)+g \varphi(t)\|_{L^{2}}^{2}+\|\varphi(t)\|_{L^{2}}^{2}+\frac{2 b d_{i}}{|d|^{2} \epsilon_{0}^{2}}|\Omega|+\|f\|_{L^{2}}^{2}+\left(1+g^{2}\right)\|h\|_{L^{2}}^{2} .
\end{gathered}
$$

So for all $t \geq t_{0}$, we see that

$$
\int_{t}^{t+1}\left\|\Lambda^{\alpha}(u+g \varphi)\right\|_{L^{2}}^{2}+\left\|\Lambda^{\alpha} \varphi\right\|_{L^{2}}^{2} d s \leq a_{1}
$$

are uniformly bounded.

Multiplying (5) by $\overline{\Lambda^{2 \alpha}(u+g \varphi)}$, then integrating over $\Omega$ and taking the real part, we have

$$
\begin{aligned}
\frac{d}{d t} \| & \Lambda^{\alpha}(u+g \varphi)\left\|_{L^{2}}^{2}-\left(\frac{g}{|d| U}+\frac{2 a d_{i}}{|d|^{2}}-\frac{2 d_{i}}{|d|^{2} U}+\beta g\right)\right\| \Lambda^{\alpha}(u+g \varphi) \|_{L^{2}}^{2} \\
& -\left(\frac{g}{|d| U}+\beta g\right)\left\|\Lambda^{\alpha} \varphi\right\|_{L^{2}}^{2} \\
\leq & -\frac{c\left(m_{i} d_{r}+m_{r} d_{i}\right)}{2|m|^{2}|d|^{2}}\left\|\Lambda^{2 \alpha}(u+g \varphi)\right\|_{L^{2}}^{2} \\
& +\operatorname{Re}\left(\frac{2 i b}{d} \int_{\Omega}|u+g \varphi|^{2}(u+g \varphi) \overline{\Lambda^{2 \alpha}(u+g \varphi)} d x\right) \\
& +\operatorname{Re}\left(\frac{2 i b}{d} \int_{\Omega}(f+g h) \overline{\Lambda^{2 \alpha}(u+g \varphi)} d x\right)
\end{aligned}
$$




$$
\begin{aligned}
\leq & -\frac{c\left(m_{i} d_{r}+m_{r} d_{i}\right)}{2|m|^{2}|d|^{2}}\left\|\Lambda^{2 \alpha}(u+g \varphi)\right\|_{L^{2}}^{2}+\frac{c\left(m_{i} d_{r}+m_{r} d_{i}\right)}{8|m|^{2}|d|^{2}}\left\|\Lambda^{2 \alpha}(u+g \varphi)\right\|_{L^{2}}^{2} \\
& +C_{2}\|u+g \varphi\|_{L^{6}}^{6}+\frac{c\left(m_{i} d_{r}+m_{r} d_{i}\right)}{4|m|^{2}|d|^{2}}\left\|\Lambda^{2 \alpha}(u+g \varphi)\right\|_{L^{2}}^{2}+C_{3}\|f+g h\|_{L^{2}}^{2} .
\end{aligned}
$$

Since

$$
\|u+g \varphi\|_{L^{6}}^{6} \leq \delta\left\|\Lambda^{2 \alpha}(u+g \varphi)\right\|_{L^{2}}^{2}+C(\delta)\|u+g \varphi\|_{L^{2}}^{\frac{6 \alpha-1}{2(3 \alpha-1)}}
$$

we can choose $\delta$ small enough such that

$$
C_{2}\|u+g \varphi\|_{L^{6}}^{6} \leq \frac{c\left(m_{i} d_{r}+m_{r} d_{i}\right)}{8|m|^{2}|d|^{2}}\left\|\Lambda^{2 \alpha}(u+g \varphi)\right\|_{L^{2}}^{2}+C_{4} .
$$

Therefore

$$
\begin{aligned}
\frac{d}{d t} \| & \Lambda^{\alpha}(u+g \varphi)\left\|_{L^{2}}^{2}-\left(\frac{g}{|d| U}+\frac{2 a d_{i}}{|d|^{2}}-\frac{2 d_{i}}{|d|^{2} U}+\beta g\right)\right\| \Lambda^{\alpha}(u+g \varphi) \|_{L^{2}}^{2} \\
& -\left(\frac{g}{|d| U}+\beta g\right)\left\|\Lambda^{\alpha} \varphi\right\|_{L^{2}}^{2} \\
& \leq C_{3}\|f+g h\|_{L^{2}}^{2}+C_{4} .
\end{aligned}
$$

Multiplying (2) by $\overline{\Lambda^{2 \alpha} \varphi}$, integrating over $\Omega$, and taking the imaginary part, we have

$$
\begin{aligned}
\frac{1}{2} \frac{d}{d t}\left\|\Lambda^{\alpha} \varphi\right\|_{L^{2}}^{2}= & -\beta\left\|\Lambda^{\alpha} \varphi\right\|_{L^{2}}^{2}+\operatorname{Re}\left(\frac{i g}{U} \int_{\Omega} u \overline{\Lambda^{2 \alpha} \varphi} d x\right)+\operatorname{Re}\left(\int_{\Omega} h(x) \overline{\Lambda^{2 \alpha} \varphi} d x\right) \\
& -\frac{m_{i}}{4|m|^{2}}\left\|\Lambda^{2 \alpha} \varphi\right\|_{L^{2}}^{2} \\
\leq & -\beta\left\|\Lambda^{\alpha} \varphi\right\|_{L^{2}}^{2}+\operatorname{Re}\left(\frac{i g}{U} \int_{\Omega}(u+g \varphi) \overline{\Lambda^{2 \alpha} \varphi} d x\right)+\frac{m_{i}}{4|m|^{2}}\left\|\Lambda^{2 \alpha} \varphi\right\|_{L^{2}}^{2} \\
& +C\|h\|_{L^{2}}^{2}-\frac{m_{i}}{4|m|^{2}}\left\|\Lambda^{2 \alpha} \varphi\right\|_{L^{2}}^{2} \\
\leq & -\beta\left\|\Lambda^{\alpha} \varphi\right\|_{L^{2}}^{2}+\frac{|g|^{2}}{2 \beta U^{2}}\left\|\Lambda^{\alpha}(u+g \varphi)\right\|_{L^{2}}^{2}+\frac{\beta}{2}\left\|\Lambda^{\alpha} \varphi\right\|_{L^{2}}^{2}+C\|h\|_{L^{2}}^{2} \\
\leq & -\frac{\beta}{2}\left\|\Lambda^{\alpha} \varphi\right\|_{L^{2}}^{2}+\frac{|g|^{2}}{2 \beta U^{2}}\left\|\Lambda^{\alpha}(u+g \varphi)\right\|_{L^{2}}^{2}+C\|h\|_{L^{2}}^{2} .
\end{aligned}
$$

Combining (16) and (17) yields

$$
\frac{d}{d t}\left(\left\|\Lambda^{\alpha}(u+g \varphi)\right\|_{L^{2}}^{2}+\left\|\Lambda^{\alpha} \varphi\right\|_{L^{2}}^{2}\right) \leq R_{2}\left(\left\|\Lambda^{\alpha}(u+g \varphi)\right\|_{L^{2}}^{2}+\left\|\Lambda^{\alpha} \varphi\right\|_{L^{2}}^{2}\right)+C_{5},
$$

where $R_{2}=\max \left\{\frac{g}{|d| U}+\frac{2 a d_{i}}{|d|^{2}}-\frac{2 d_{i}}{|d|^{2} U}+\beta g+\frac{g^{2}}{\beta U^{2}}, \frac{g}{|d| U}+\beta g-\beta\right\}$.

Since

$$
\int_{t}^{t+1} R_{2} d s \leq R_{2}, \quad \int_{t}^{t+1} C_{5} d s \leq a_{2}
$$


applying the uniform Gronwall's inequality (Lemma 7), we have

$$
\left\|\Lambda^{\alpha}(u+g \varphi)\right\|_{L^{2}}^{2}+\left\|\Lambda^{\alpha} \varphi\right\|_{L^{2}}^{2} \leq\left(a_{1}+a_{2}\right) e^{R_{2}}, \quad t \geq t_{0}+1 .
$$

The above inequality can ensure us the existence of an absorbing ball in $H^{\alpha} \times H^{\alpha}$. In fact, let $\mathcal{B}$ be a bounded set in $H^{\alpha} \times H^{\alpha}$. Obviously, it is also a bounded set in $L^{2} \times L^{2}$ and $S(t) \mathcal{B} \subset \mathcal{B}_{0}$ for $t \geq t_{0}$. From (18), it follows that $S(t) \mathcal{B} \subset \mathcal{B}_{1}$, where $\mathcal{B}_{1}=B\left(0, \rho_{1}\right)$ is a ball with radius $\rho_{1}^{2}=\rho_{0}^{2}+\left(a_{1}+a_{2}\right) e^{R_{2}}$ in $H^{\alpha} \times H^{\alpha}$. Since the embedding $H^{\alpha} \times H^{\alpha} \hookrightarrow L^{2} \times L^{2}$ is compact, we obtain

$$
\bigcup_{t \geq t_{0}+1} S(t) \mathcal{B} \text { is relatively compact in } L^{2} \times L^{2}
$$

Thus the assertion of item (iii) has been proved.

Notice that items (i) and (ii) can be verified similar to the proof of Lemmas 1-4 and item (iv) is a direct corollary of items (i)-(iii). Thus the proof is completed.

\section{Competing interests}

The authors declare that they have no competing interests.

\section{Authors' contributions}

The authors contributed equally to the writing of this paper. All authors read and approved the final manuscript.

\section{Acknowledgements}

This work was supported by the National Natural Science Foundation of China (Grant No. 11271141; No. 11101160; No.

11426069; No. 61375006)

Received: 23 July 2016 Accepted: 13 December 2016 Published online: 10 January 2017

\section{References}

1. Machida, M, Koyama, T: Time-dependent Ginzburg-Landau theory for atomic Fermi gases near the BCS-BEC crossover. Phys. Rev. A 74, 033603 (2006)

2. Chen, S, Guo, B: On the Cauchy problem of the Ginzburg-Landau equations for atomic Fermi gases near the BCS-BEC crossover. J. Partial Differ. Equ. 22(3), 218-233 (2009)

3. Chen, S, Guo, B: Existence of the weak solution of coupled time-dependent Ginzburg-Landau equations. J. Math. Phys. 51(3), 033507 (2010)

4. Chen, S, Guo, B: Classical solutions of time-dependent Ginzburg-Landau theory for atomic Fermi gases near the BCS-BEC crossover. J. Differ. Equ. 251, 1415-1427 (2011)

5. Fang, S, Jin, L, Guo, B: Global attractor for the initial-boundary value problems for Ginzburg-Landau equations for atomic Fermi gases near the BCS-BEC crossover. Nonlinear Anal. 72, 4063-4070 (2010)

6. Guo, C, Chen, R, Fang, S, Wang, X: Finite difference method for Ginzburg-Landau equations for atomic Fermi gases near the BCS-BEC crossover. In: World Congress on Engineering and Technology, pp. 526-529 (2011)

7. Guan, J, Fang, S, Wang, X, Guo, C: Hopf bifurcations of traveling wave solutions for time-dependent Ginzburg-Landau equation for atomic Fermi gases near the BCS-BEC crossover. Commun. Nonlinear Sci. Numer. Simul. 18, 124-135 (2013)

8. Pu, X, Guo, B: Well-posedness and dynamics for the fractional Ginzburg-Landau equation. Appl. Anal. 1, 1-17 (2011)

9. Guo, B, Han, Y, Xin, J: Existence of the global smooth solution to the period boundary value problem of fractional nonlinear Schrödinger equation. Appl. Math. Comput. 204, 468-477 (2008)

10. $\mathrm{Pu}, \mathrm{X}, \mathrm{Guo}, \mathrm{B}$ : The fractional Landau-Lifshitz-Gilbert equation and the heat flow of harmonic maps. Calc. Var. 42, 1-19 (2011)

11. Pu, X, Guo, B: Well-posedness for the fractional Landau-Lifshitz equation without Gilbert damping. Calc. Var. 46, 441-460 (2013)

12. Tarasov, V: Fractional Dynamics: Applications of Fractional Calculus to Dynamics of Particles, Fields and Media. Springer, Berlin (2010)

13. Kilbas, AA, Srivastava, HM, Trujillo, JJ: Theory and Applications of Fractional Differential Equations. Elsevier, Amsterdam (2006)

14. Podlubny, I: Fractional Differential Equations. Academic Press, San Diego (1999)

15. Miller, KS, Ross, B: An Introduction to the Fractional Calculus and Fractional Differential Equations. Wiley, New York (1993)

16. Temam, R: Infinite-Dimensional Dynamical Systems in Mechanics and Physics. Springer, New York (1998) 